Cahiers
de a Recherche
surles Droits

Cahiers de la recherche sur les droits

fondamentaux

$6 \mid 2008$

Pouvoirs exceptionnels et droits fondamentaux

\title{
Les implications nouvelles du principe de sécurité juridique en droit administratif
}

Note sous CE, Ass., 16 juillet 2007, Société Tropic Travaux Signalisation

Fabien Bottini

\section{CpenEdition}

\section{Journals}

Édition électronique

URL : https://journals.openedition.org/crdf/6912

DOI : $10.4000 /$ crdf.6912

ISSN : 2264-1246

Éditeur

Presses universitaires de Caen

Édition imprimée

Date de publication : 31 décembre 2008

Pagination : 161-172

ISBN : 978-2-84133-259-5

ISSN : 1634-8842

\section{Référence électronique}

Fabien Bottini, «Les implications nouvelles du principe de sécurité juridique en droit administratif », Cahiers de la recherche sur les droits fondamentaux [En ligne], 6 | 2008, mis en ligne le 11 décembre 2020, consulté le 14 novembre 2022. URL : http://journals.openedition.org/crdf/6912 ; DOI : https:// doi.org/10.4000/crdf.6912 


\title{
Les implications nouvelles du principe de sécurité juridique en droit administratif
}

\author{
Note sous CE, Ass., 16 juillet 2007, \\ Société Tropic Travaux Signalisation
}

\author{
Fabien BOTTINI \\ Docteur en droit public \\ Chargé de cours à l'Université du Havre
}

I. La sécurité juridique, facteur d'un renforcement des garanties offertes aux justiciables

A. La reconnaissance du droit des justiciables de ne pas voir leur situation bouleversée par un changement de jurisprudence

B. La reconnaissance d'un droit d'action aux concurrents évincés devant le juge de plein contentieux

II. La sécurité juridique, facteur d'un accroissement des pouvoirs du juge

A. La reconnaissance du pouvoir des juridictions suprêmes de moduler dans le temps les effets de leurs revirements de jurisprudence

B. La reconnaissance du pouvoir du juge de plein contentieux de moduler les effets de l'annulation d'un contrat administratif

Annexe

Résumé: Quelles sont les conséquences de la consécration du principe de sécurité juridique en droit interne au regard de la validité des contrats administratifs et des revirements de jurisprudence? Telle était la question qui se posait en l'espèce à l'assemblée du Conseil d'État.

La haute juridiction y répond en opérant une double modification de jurisprudence. La première le conduit à simplifier l'état du droit en ouvrant aux concurrents évincés de la conclusion d'un contrat administratif de nouvelles voies de recours. En même temps qu'il leur permet de demander au juge des référés d'ordonner la suspension de son exécution, il les autorise en effet à contester sa validité directement devant le juge de plein contentieux après sa signature. Le second revirement est tout aussi spectaculaire, sinon plus : dans la mesure où le Conseil profite de l'affaire qui lui est soumise pour se reconnaître le droit de moduler dans le temps les effets de ses changements de jurisprudence.

Cet arrêt est intéressant en ce qu'il illustre les implications nouvelles de la consécration du principe de sécurité juridique en droit administratif, celle-ci semblant conduire les magistrats à renforcer les garanties offertes aux justiciables contre l'instabilité du droit en accroissant leurs pouvoirs. 
Si, en 1790, Robespierre s'écriait: «ce mot de jurisprudence $[. .$.$] doit être effacé de notre langue » { }^{1}$, l'expression semble encore avoir de beaux jours devant elle, comme l'illustre une décision de l'assemblée plénière du Conseil d'État en date du 16 juillet 2007.

Les faits à l'origine de cette affaire sont assez simples: le marquage des aires d'avions et des chaussées routières de l'aéroport Le Raizet de Pointe-à-Pitre ayant besoin d'un nouveau coup de pinceau, la Chambre de commerce et d'industrie locale faisait un appel d'offres pour attribuer le marché. La Société Tropic Travaux Signalisation concourait à son obtention. Mais, le 14 novembre 2005, la CCI rejetait sa candidature au profit de l'entreprise Rugoway avec qui elle signait le contrat.

Contestant la régularité tant des actes détachables préalables à la passation du marché que la régularité du marché lui-même, la Société Tropic Travaux Signalisation demandait au juge des référés du tribunal administratif de Basse-Terre d'ordonner la suspension de leur exécution, sur le fondement de l'article L. 521-1 du Code de la justice administrative. Le juge des référés ayant rejeté ses prétentions par ordonnance du 2 mars 2006, la société demandait au Conseil d'État d'annuler sa décision et d'ordonner les suspensions demandées, par requête enregistrée au secrétariat du contentieux le 21 mars 2006.

À l'appui de son recours, la société invoquait notamment un détournement de pouvoir, propre selon elle à entacher d'irrégularité le marché.

Quelles sont les conséquences de la consécration de l'exigence de sécurité juridique en droit interne au regard de la validité des contrats administratifs et des revirements de jurisprudence? Telle était la question qui se posait en l'espèce à la haute juridiction.

Dans sa décision, le Conseil d'État commence par annuler l'ordonnance du juge du fond, après avoir constaté qu'il avait commis une erreur de droit en déboutant la partie demanderesse sans vérifier si elle s'était bien portée candidate à l'attribution du marché litigieux. Ayant évoqué l'affaire, par application de l'article L. 821-2 du Code de justice administrative, le juge suprême conclut cependant au rejet de la demande, motif pris de ce que la requérante ne soulevait pas de moyens sérieux. Mais il le fait après avoir opéré un double revirement de jurisprudence. Le premier ouvre aux concurrents évincés de la conclusion d'un contrat administratif de nouvelles voies de recours. Non content de les autoriser à contester la validité du contrat directement devant le juge de plein contentieux après sa signature, il leur permet d'assortir leur recours d'un référé suspensif tendant à voir prononcer la suspension de l'exécution de la convention, à titre conservatoire. Le second revirement est tout aussi spectaculaire, sinon plus : puisque le Conseil profite de la présente espèce pour se reconnaître le droit de moduler dans le temps les effets de ses changements de jurisprudence.

Cet arrêt est donc intéressant en ce qu'il illustre les implications nouvelles de la consécration du principe de sécurité juridique en droit interne, celle-ci encourageant les magistrats à renforcer les garanties offertes aux justiciables contre l'instabilité juridique (I) en accroissant leurs pouvoirs (II).

\section{La sécurité juridique, facteur d'un renforcement des garanties offertes aux justiciables}

La consécration du principe de sécurité juridique a en l'espèce conduit le Conseil d'État à renforcer les garanties offertes aux justiciables. Tout d'abord, en leur reconnaissant le droit de ne pas voir leurs situations légalement acquises brutalement bouleversées par un revirement de jurisprudence (A). Ensuite, en ouvrant aux concurrents évincés de la passation d'un contrat administratif de nouvelles voies de recours de façon à simplifier l'état du droit (B).

\section{A. La reconnaissance du droit des justiciables de ne pas voir leur situation bouleversée par un changement de jurisprudence}

En l'espèce, le Conseil d'État considère qu'eu égard à l'impératif de sécurité juridique tenant à ce qu'il ne soit pas porté une atteinte excessive aux situations légalement acquises, un revirement de jurisprudence n'est pas nécessairement applicable à celles qui lui sont antérieures.

Ce faisant, il exprime sa volonté de s'aligner sur les jurisprudences européennes ${ }^{2}$ et judiciaire afin de renforcer les garanties offertes aux justiciables contre l'instabilité du droit.

En ce qui la concerne, la Cour de cassation considérait jusqu'en 2004 que les revirements étaient par essence rétroactifs ${ }^{3}$. La rigueur de cette solution apparaissait toutefois sévère eu égard aux règles applicables en droit européen et dans certains pays étrangers, dans les pays anglosaxons notamment où le principe du prospective overrulings permet aux tribunaux de limiter leurs revirements, par exemple aux litiges à naître ${ }^{4}$.

1. "Intervention de Maximilien Robespierre le 18 novembre 1790, lors de la discussion sur le tribunal de cassation », Arch. parlem., t. XIX, p. 516.

2. Voir, par ex., CEDH, 13 juin 1979, Marcks c / Belgique; CJCE, 27 février 1985, Aff. 112 / 83, rec., p. 719, pts 16-18.

3. Cass., civ., $1^{\text {re }}, 21$ mars 2000, D., 2000, jur., p. 593, note C. Atias; civ., $1^{\text {re }}, 9$ octobre 2001, D., jur., p. 3470, note D. Thouvenin ; civ., $2^{\mathrm{e}}, 2$ octobre $2002, D_{\text {. }}$ 2003, jur., p. 513, note C. Atias; soc., 25 juin 2003, D., 2004, jur., p. 1761, note M. Julien.

4. US S. Ct, 5 décembre 1932, Great Northern Railway Co v. Sunburst Oil \& Refining Co (287 S. Ct 358). Sur cette question, voir C. Mouly, « Rapport sur les revirements de jurisprudence de la Cour de cassation ", in L'Image doctrinale de la Cour de cassation, Paris, La Documentation française, 1994, p. 139141 ; H. Muir Watt, «La gestion de la rétroactivité des revirements de jurisprudence : systèmes de common Law », in Les Revirements de jurisprudence : rapport remis à Monsieur le Premier Président Guy Canivet, mardi 30 novembre 2004, N. Molfessis (dir.), Paris, Litec, 2005, p. 53-71; F. Dieu, « La modulation des effets des annulations contentieuses ou comment concilier principe de légalité et principe de sécurité juridique», AJDA, 2006.2428. Dans le même ordre d'idées, on peut relever que les cours constitutionnelles autrichienne, allemande, italienne et portugaise limitent parfois la rétroactivité de 
Mais la Cour invoquait deux considérations à son appui. La première consistait à dire que «l'interprétation jurisprudentielle d'une même norme à un moment donné ne $\mathrm{p}$ [ouvait] être différente selon l'époque des faits considérés». Quant à la seconde, elle postulait que «nul ne p[ouvait] se prévaloir d'un droit acquis à une jurisprudence figée $»^{5}$.

Chacune de ces justifications apparaissait pourtant contestable. Il était d'abord discutable d'affirmer que l'interprétation d'une norme ne peut changer dans le temps, puisqu'il suffit de feuilleter les recueils d'arrêts et de comparer la solution donnée à un même problème juridique sur le fondement d'un même texte à quelques années d'intervalle pour se rendre compte qu'elle varie par essence. S'il en était autrement, le problème des modifications de jurisprudence ne se poserait d'ailleurs pas, dans la mesure où il n'y aurait par définition pas de revirements. L'argument selon lequel les justiciables n'étaient pas fondés à se prévaloir de la jurisprudence antérieure était tout aussi polémique, dès lors que la connaissance de cette règle avait pu seule les déterminer à agir.

Diverses décisions de justice mettaient en outre en lumière le caractère injuste de la solution traditionnelle. Tel était par exemple le cas de l'arrêt de la Cour de cassation condamnant civilement un médecin pour n'avoir pas informé son patient des risques exceptionnels qu'il encourait, alors même qu'à l'époque des faits la jurisprudence considérait qu'il ne commettait pas de faute en s'abstenant de le faire ${ }^{6}$; ou encore de la décision de la chambre sociale de la Cour de cassation de revenir sur son refus de considérer l'existence d'une contrepartie financière comme nécessaire à la validité de l'obligation de nonconcurrence postcontractuelle ${ }^{7}$.

Ces affaires produisaient un remous tel au sein du corps médical et des acteurs économiques qu'elles conduisaient Guy Canivet, le premier président de la Cour de cassation, à demander à un groupe de travail présidé par le professeur Molfessis de réfléchir à la façon de rendre les revirements davantage compatibles avec les attentes des justiciables.

Dans un rapport remis à son commanditaire le 30 novembre 2004, ses membres s'inspiraient d'une décision précédente de la Cour ${ }^{8}$ pour lui suggérer de moduler dans le temps les effets de ses changements d'interprétation, afin d'assurer une meilleure conciliation de l'intérêt général et des droits acquis des particuliers ${ }^{9}$. La faculté du juge de leur imposer le respect d'obligations qu'ils ne pouvaient pas connaître au moment où ils avaient agi leur apparaissait en effet contraire au principe de sécurité juridique que la haute juridiction avait consacré en $2003^{10}$.

«Nul n'étant censé ignoré la loi », ce principe impose de faire en sorte que chacun soit « en mesure de déterminer ce qui est permis » ou « défendu par le droit applicable ${ }^{11}$. C'est pourquoi le juge constitutionnel le rattache à la garantie des droits de l'article 16 de la Déclaration des droits de l'homme et du citoyen ${ }^{12}$, et pourquoi il revêt une double dimension ${ }^{13}$. Une dimension objective d'une part, qui implique de faire en sorte que les normes édictées soient claires, intelligibles et stables. Une dimension subjective d'autre part, qui commande de respecter le droit de chacun au maintien de la situation juridique qu'il a légalement acquise, au besoin en assortissant de mesures transitoires les modifications brutales de l'état du droit. Dans ce dernier aspect, le principe de sécurité juridique revêt la forme d'un principe dit de confiance légitime, que l'on peut définir comme «la confiance que les destinataires de règles ou de décisions de l'État sont normalement en droit d'avoir dans la stabilité, du moins pour un certain temps, des situations établies sur la base de ces règles ou de ces décisions ${ }^{14}$.

Or, le rapport Molfessis encourageait les juges suprêmes à tirer toutes les conséquences de la consécration du principe de sécurité juridique en droit privé en confortant la jurisprudence par laquelle ils s'étaient reconnus le

leurs déclarations d'inconstitutionnalité (O. Dubos, F. Melleray, «La modulation dans le temps des effets de l'annulation d'un acte administratif», $D A, 2004$, chron. $\left.\mathrm{n}^{\circ} 15\right)$.

5. Cass., 9 octobre 2001, D., jur., p. 3470 .

6. Ibid.

7. Cass., soc., 10 juillet 2002, MM. Moline, Salembier et Barbier (3 esp.), BICC, 15 septembre 2002, nº 562, avis de M. Kehrig.

8. Cass., civ., $2^{\text {e }}, 8$ juillet 2004, D., 2004, jur., p. 2956, note C. Bigot.

9. Les Revirements de jurisprudence..., p. 48-50. Voir également C. Mouly, « Rapport sur les revirements de jurisprudence... », p. 123-148 ; « Le revirement pour l'avenir ", JCP G, 1996, I.3776; P. Sargos, «L'horreur économique dans la relation de droit (libres propos sur le "Rapport sur les revirements de jurisprudence") », Dr. soc., 2005.123.

10. Cass., civ., $2^{\mathrm{e}}$, 30 janvier 2003, Fillaud, D., 2003, jur., p. 2722, note E. Ben Merzouk.

11. «EDCE », 2006, $\mathrm{n}^{\circ}$ 57, p. 281. Dans le même sens, voir S. Calmes, «Sécurité juridique et droits fondamentaux », in Valeurs républicaines et droits fondamentaux de la personne humaine en 2003 et 2004, G. Lebreton (dir.), Paris, L'Harmattan, 2006, p. 148: «la sécurité juridique est la qualité d'un ordre juridique qui assure au citoyen lisibilité et confiance dans ce qui constitue le droit en un moment donné et ce qui, selon toute probabilité, sera le droit dans l'avenir"».

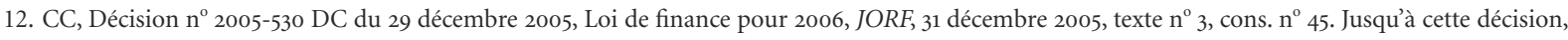
certains auteurs faisaient dériver le principe de sécurité juridique du droit à la «sûreté " (Déclaration des droits de l'homme et du citoyen, art. 2). En ce sens, voir F. Luchaire, La Protection constitutionnelle des droits et libertés, Paris, Économica, 1987, p. 42-43: l'auteur constate que «le principe du respect [...] des situations légalement acquises » se rattache à « la sûreté» dans la mesure où « la sûreté consiste dans la protection accordée par la société à chaque citoyen pour la conservation de [...] ses droits ». D'autres auteurs estimaient toutefois que la jurisprudence constitutionnelle rattachait indirectement la sécurité juridique à «la garantie des droits requise par »l' «article 16 » de la Déclaration des droits de l'homme et du citoyen, « et plus spécialement [d] es droits de la défense» (D. Chagnollaud, "Sécurité juridique (Droit à la) », in Dictionnaire des droits fondamentaux, D. Chagnollaud, G. Drago (dir.), Paris, Dalloz, 2006, p. 677). Voir, estimant les deux fondements possibles, L. Favoreu et al., Droit des libertés fondamentales, Paris, Dalloz, 2007, n 393 ; S. Calmes, « Sécurité juridique et droits fondamentaux », p. 142.

13. Sur ce point, voir M. Fromont, «Le principe de sécurité juridique», AJDA, 1996, nº spécial, p. 178.

14. Ibid., p. 179 ; P. Cassia, «La sécurité juridique, un "nouveau” principe général du droit aux multiples facettes », D., 2006, chr., p. 1190. 
droit de limiter dans le temps les effets de certains de leurs revirements. C'est ainsi que depuis 2004 la haute juridiction se reconnaît la possibilité de rendre une modification d'interprétation inapplicable aux litiges pendants ${ }^{15}$.

Sa prise de position posait en filigrane la question de l'attitude du Conseil d'État. La juridiction suprême de l'ordre administratif allait-elle se rallier à son homologue judiciaire?

Jusqu'au prononcé de la décision commentée, le Conseil s'interdisait de franchir le « Rubicon ${ }^{16}$. C' est du moins ce qui ressortait de deux arrêts respectivement rendus en $2004^{17}$ et 2005 . À cette date, le juge administratif suprême refusait en effet de suivre son commissaire du gouvernement qui l'invitait à rendre inapplicable un revirement de jurisprudence ${ }^{18}$ aux actions qui lui étaient antérieures, manifestant ainsi son attachement à la solution classique ${ }^{19}$.

La position des juges du palais Royal semblait liée à leur refus de reconnaître le principe de sécurité juridique. Bien que nombre de ses conséquences y soient depuis longtemps admises, ce dernier a en effet longtemps constitué un principe "clandestin ${ }^{20}$ du droit administratif. Guider l'action de la jurisprudence, notamment au travers des principes de non-rétroactivité des actes unilatéraux et de l'intangibilité des actes créateurs de droit ${ }^{21}$, sans jamais être expressément visé par le Conseil : tel semblait devoir être son destin jusqu'au milieu des années 1990. Si, à cette date, la haute juridiction a commencé à reprendre l'expression «sécurité juridique» à son compte, c'était pour affirmer que le principe même n'était pas invocable en dehors de la mise en œuvre du droit communautaire par les autorités françaises ${ }^{22}$; ou simplement pour rappeler quel était l'objectif poursuivi par le législateur lorsqu'il avait voté la loi applicable au litige ${ }^{23}$.

Sa consécration en droit administratif était cependant annoncée par celle de certaines de ses implications. Elle l'était tout d'abord par la décision de la haute juridiction de reconnaître l'existence du principe de confiance légitime - bien que cette reconnaissance ne s'étende pas en dehors des situations « régie[s] par le droit communautaire ${ }^{24}$. Elle l'était ensuite par sa décision de circonscrire davantage la possibilité de l'administration de retirer un acte administratif individuel créateur de droit illégal ${ }^{25}$. Enfin, elle l'était par sa décision de limiter les effets dans le temps de l'annulation d'un acte administratif unilaté$\mathrm{ral}^{26}$.

Si la consécration du principe de sécurité juridique par le Conseil d'État ${ }^{27}$ n'a donc pas été une surprise - ce dernier prenant soin de rappeler en l'espèce qu'il s'agit d'un «impératif» et non d'un simple «objectif» - les affaires «Ternon» et «AC! » posaient en toile de fond la question de la possibilité de moduler dans le temps les effets des revirements de la jurisprudence administrative $^{28}$. Dès lors en effet que de tels changements d'interprétation peuvent compromettre la stabilité du droit autant que le retrait ou l'annulation d'un acte administratif unilatéral, ne pas revoir la solution traditionnelle en la matière au regard de l'exigence nouvelle de sécurité juridique exposait le juge administratif au risque de se voir reprocher d'imposer aux autres des obligations qu'il ne respecte pas, sur le mode du «faites ce que dis, pas ce que je fais ». D'autant que l'état du droit se heurtait au désir de plus en plus grand des justiciables de voir « sécuriser les interprétations jurisprudentielles ${ }^{29}$.

De ce point de vue, la consécration de ce principe semble avoir encouragé le Conseil à profiter de la présente affaire pour franchir le pas, puisqu'il se reconnaît en l'espèce le droit de moduler la portée temporelle de ses changements d'interprétation, afin de renforcer les garanties offertes aux particuliers contre l'instabilité du droit. La présente décision illustre ainsi sa volonté de se rallier à la jurisprudence européenne et à celle de la Cour de cassation, en se reconnaissant la faculté de limiter les

15. Cass., soc., 17 décembre 2004, société Samse, D., 2004, IR, p. 110 ; Ass. plén., 21 décembre 2006, société La Provence, $B I C C, 1^{\mathrm{er}}$ mars $2007, \mathrm{n}^{\mathrm{0}}$ 656, p. 28 sq., rapport de M. Lacabaras, avis de M. Legoux; Ass. plén., 21 décembre 2006, société La Briocherie et société Centea (2 esp.), ibid., p. 50 sq., rapport de M. Loriferne, avis de M. de Gouttes.

16. P. Morvan, «Le revirement de jurisprudence pour l'avenir. Humble adresse aux magistrats ayant franchi le Rubicon », D., 2005, doct., p. 247.

17. CE, 14 juin 2004, SCI Saint-Lazare, DA, 2004, $n^{\circ} 166$, note Noguellou.

18. CE, 28 septembre 2005, Louis, AJDA, 2006.103, note A.-M. Mazetier. Le Conseil d’État ayant estimé que le recours administratif préalable était ouvert à des individus non visés par le texte qui l'institue, son commissaire du gouvernement l'invitait en vain à ne pas appliquer cette solution aux litiges portés en justice avant qu'elle ne soit rendue.

19. CE, Sect., 10 mars 2006, Leroy-Merlin, AJDA, 2006.681, note B. Seiller.

20. B. Mathieu, «La sécurité juridique, un principe constitutionnel clandestin mais efficient», in Mélanges Patrice Gélard, Paris, Montchrestien, 20oo, p. 301 ; «Le principe de sécurité juridique entre au Conseil d'État», AJDA, 2006.841.

21. En ce sens, voir B. Pacteau, «La sécurité juridique, un principe qui nous manque?», AJDA, 1995, n spécial, p. 151; M. Fromont «Le principe de sécurité juridique », p. 178 .

22. CE, 28 mai 1997, Macabeo, req. n 180 726, inédit; 30 décembre 1998, soc. Entreprise Chagnaud SA.

23. CE, 29 novembre 1999, Laurent, req. $n^{\circ} 171793$, inédit.

24. CE, 16 mars 1998, Association des élèves, parents d'élèves et professeurs des classes préparatoires vétérinaires et $\mathrm{M}^{\text {lle }}$ Pujol, rec., p. 84 ; Ass., 11 juillet 2001, FNSEA et al., RFDA, 2002.33, concl. F. Séners.

25. CE, Ass., 26 octobre 2001, Ternon, AJDA, 2001.1034, chron. M. Guyomar et P. Collin : le délai de retrait est désormais de quatre mois à compter de l'édiction de l'acte.

26. CE, Ass., 11 mai 2004, Association AC!, AJDA, 2004.1183, note C. Landais et F. Lenica; 11 janvier 2006, AVFS, AJDA, 2006.116.

27. CE, Ass., 24 mars 2006, KPMG, AJDA, 2006.1028, chron. C. Landais et F. Lenica; RFDA, 2006.463, concl. Y. Aguila. Encore que certains s'interrogent sur la réalité de cette consécration par le juge administratif. Voir F. Melleray, «L'arrêt KPMG consacre-t-il vraiment le principe de sécurité juridique? », AJDA, 2006.897 : l'auteur se demande si en fait « d'un principe général de sécurité juridique », le Conseil d'État n'a pas consacré « un principe de confiance légitime "à la française" ".

28. Sur cette question, voir W. Dross, «La jurisprudence est-elle seulement rétroactive?», D., 2006, chr., p. 472.

29. G. Canivet, «Incertitude et politique jurisprudentielle de sécurisation du droit », JCP G, $2005, \mathrm{n}^{\circ}$ 4, p. XX, $\mathrm{n}^{\circ} 15$. 
désagréments que de tels changements peuvent causer aux parties. De sorte que, si la modification apportée à l'interprétation traditionnelle d'une règle de droit demeure en principe rétroactive, ce principe n'est désormais plus absolu. Ainsi, tandis qu'auparavant «la sécurité juridique se trouv[ait] [...] nécessairement sacrifiée au progrès présumé du droit ${ }^{30}$, ledit progrès passe désormais par une meilleure prise en compte des aspirations des citoyens à un plus grand respect de ce principe.

C'est encore la reconnaissance de cet « impératif » qui semble avoir conduit le juge administratif à profiter de l'affaire qui lui était soumise pour simplifier l'état du droit, en ouvrant de nouvelles voies d'action aux concurrents évincés de la passation d'un contrat administratif, notamment devant le juge de plein contentieux.

\section{B. La reconnaissance d'un droit d'action aux concurrents évincés devant le juge de plein contentieux}

La consécration du principe de sécurité juridique a encouragé le Conseil d'État à supprimer la possibilité jusqu'à présent offerte aux concurrents évincés de la signature du contrat de demander l'annulation pour excès de pouvoir des actes préalables qui en sont détachables. En contrepartie, le droit de chacun à un recours effectif l'a incité à reconnaître auxdits concurrents la faculté de former un recours de pleine juridiction à l'encontre de la convention - ou de celles de ses clauses qui en sont divisibles -, ledit recours pouvant éventuellement être assorti d'une demande tendant à voir le juge des référés ordonner la suspension de l'exécution du contrat.

Ce faisant, la haute juridiction renverse totalement la solution traditionnelle. Postulant que seules les parties au contrat sont fondées à en contester la validité, l'état du droit dénie en effet en principe aux tiers la possibilité de saisir le juge de plein contentieux d'une action en nullité ${ }^{31}$; tandis que «l'exception de recours parallèle» le conduit simultanément à leur refuser celle de contester la légalité du contrat par le biais du recours pour excès de pouvoir $(\mathrm{REP})^{32}$. Les personnes extérieures à la convention ne sont ainsi en principe recevables qu'à contester les actes préalables qui s'en détachent devant le juge de l'excès de pou- voir $^{33}$. Constituent de tels actes, ceux qui, comme dans la présente affaire, se rapportent à la passation du contrat, telles que la décision de l'administration d'évincer un candidat de l'obtention d'un marché public, d'attribuer ledit marché à une entreprise concurrente ou encore celle de conclure l'accord avec elle ${ }^{34}$. Bien que l'apparente rigueur de cette solution conduise le Conseil d'État à la maintenir depuis près d'un siècle, deux séries de reproches peuvent toutefois lui être adressées.

D'abord, sa logique n'est pas implacable, dans la mesure où elle repose sur l'idée que seules les parties au contrat peuvent avoir un intérêt à son annulation alors que les tiers peuvent également y trouver avantage. Le législateur et la jurisprudence en ont d'ailleurs convenu. Le premier a ainsi autorisé les préfets à contester la légalité des engagements passés par les collectivités locales par le biais du déféré préfectoral ${ }^{35}$ qui, juridiquement, s'analyse comme un $\mathrm{REP}^{36}$; et reconnu aux contribuables communaux le droit d'agir en lieu et place de leur collectivité ${ }^{37}$. Pour sa part, la jurisprudence administrative l'a admis en ouvrant aux tiers le droit de demander l'annulation des contrats d'embauche des agents non titulaires des collectivités territoriales ${ }^{38}$; ou celle des clauses réglementaires qui sont divisibles des clauses proprement contractuelles d'un contrat administratif ${ }^{39}$.

Si ces concessions sont autant de remises en cause de la jurisprudence administrative traditionnelle, celle-ci paraît d'autant plus contestable que l'artifice de la théorie des actes détachables permet en principe aux tiers d'obtenir l'annulation d'un contrat dont les dispositions sont pourtant parfaitement valables. Sans doute le Conseil d'État a-t-il dénié un effet utile à l'invalidation des actes détachables quant à la régularité même de la convention jusque dans les années 1990, l'intéressé ne se lassant pas d'affirmer que «l'annulation par le juge de l'excès de pouvoir, à la demande d'un tiers, d'un » tel «acte [...], n'a par elle-même aucun effet direct sur [1] e contrat ${ }^{40}$. Mais les choses ont changé dans la dernière décennie du $\mathrm{XX}^{\mathrm{e}}$ siècle. D'abord, parce que la jurisprudence oblige depuis lors le juge de plein contentieux, saisi par l'une des parties, à tirer d'office les conséquences de l'annulation d'un acte détachable prononcée par le juge de l'excès de pouvoir, quant à l'existence de la convention ${ }^{41}$. Ensuite, parce que

30. J. Rivero, «Sur la rétroactivité de la règle jurisprudentielle », AJDA, 1968.16.

31. CE, 24 décembre 1897, Le Buf, rec., p. 848 ; Sect., 17 janvier 1936, Ligue des consommateurs d'électricité et ville de Tonneins, rec., p. 76 ; Sect., 20 janvier 1978, Syndicat national de l'enseignement technique agricole public, rec., p. 22.

32. CE, 5 avril 1940, Sieurs Gay, Vistel et al., rec., p. 136; Sect., 30 juin 1944, Sieur Pazery, rec., p. 185 ; Ass., 16 avril 1986, Compagnie luxembourgeoise de télédiffusion et al., AJDA, 1986.284, chron. M. Azibert et M. Fornacciari.

33. CE, 11 décembre 1903, Commune de Gorre, rec., p. 771; 29 décembre 1905, Martin, RDP, 1906.249, note G. Jèze, concl. Romieu.

34. CE, Sect., 9 novembre 1934, Chambre de commerce, d'industrie et d'agriculture de Tamatave, rec., p. 1034; Sect., 7 février 1936, Département de la Creuse, D., 1937.3.23, note C. Blaevoet.

35. L. modifiée $\mathrm{n}^{\circ}$ 82-213 du 2 mars 1982, relative aux droits et libertés des communes, des départements et des régions, art. 3 codifié à l'article L. $2131-6$ du CGCT; CE, 4 novembre 1994, Département de la Sarthe, rec., p. 801.

36. CE, Sect., 26 juillet 1991, Commune de Sainte-Marie (Réunion), AJDA, 1991.693, chron. C. Maugüé et R. Schwartz.

37. Code des communes, art. L. 316-5 repris à l'article L. 2132-5 du CGCT. Pour un exemple d'application, voir CE Sect. 29 novembre 1929, Sieurs Chatelot et al., rec., p. 1049.

38. CE, 30 octobre 1998, Ville de Lisieux, AJDA, 1998.969, chron. F. Raynaud et P. Fombeur.

39. CE, Ass., 10 juillet 1996, Cayzeele, AJDA, 1996.732, chron. D. Chauvaux et T.-X. Girardot.

40. EDCE, $1989, \mathrm{n}^{\circ} 41$, p. 127.

41. CE, $1^{\text {er }}$ octobre 1993, Société Le Yacht-Club international de Bormes-les-Mimosas, AJDA, 1993.810. 
le Conseil d'État contraint l'administration à saisir le juge du contrat, afin de voir prononcer sa résolution, chaque fois que la décision de le passer s'est trouvée annulée pour excès de pouvoir ${ }^{42}$. Enfin, parce que le législateur a parallèlement reconnu aux tiers ayant obtenu du juge de l'excès de pouvoir l'annulation d'un acte détachable la faculté de demander au juge de l'exécution qu'il enjoigne aux cocontractants de saisir le juge du contrat, afin qu'il prononce la nullité de la convention ${ }^{43}$, éventuellement sous astreinte ${ }^{44}$. En présence de telles solutions, le refus de la haute juridiction de reconnaître un droit d'action direct aux tiers apparaît donc discutable, tant il complique inutilement l'état du droit. La voie de recours qui leur est ouverte est tellement lourde et compliquée qu'elle contrevient à l'exigence de clarté et d'accessibilité de la règle de droit dérivée de l'exigence de sécurité juridique. Elle méconnaît en outre l'impératif de stabilité des rapports juridiques qui en découle, dès lors que le droit positif peut conduire à cette situation paradoxale qu'un contrat en soi valable se trouve malgré tout invalidé ${ }^{45}$.

L'arrêt commenté montre toutefois que cette complexification de la règle de droit n'est qu'un préalable à sa simplification. Car les solutions précitées semblent rétrospectivement participer d'une politique jurisprudentielle des «petits pas ${ }^{46}$ tendant à tirer les conséquences des aspirations des justiciables à une plus grande sécurité juridique de manière prudente, de façon à ne pas compromettre la stabilité des relations contractuelles. Si, de ce point de vue, la décision marque un jalon important de cette évolution, elle l'opère en conciliant deux aspects en l'espèce contradictoires de la garantie des droits affirmée à l'article 16 de la Déclaration des droits de l'homme et du citoyen.

D’un côté, la consécration du principe de sécurité juridique en droit interne a conduit le juge à supprimer la faculté offerte aux tiers évincés de la passation du contrat de contester les actes qui s'en détachent après sa signature ${ }^{47}$. Jusqu'à ce que l'arrêt commenté soit rendu en effet, les actes détachables non publiés pouvaient en principe être contestés à n'importe quel moment devant le juge de l'excès de pouvoir, puisque, à défaut de publication, les délais de recours n'avaient en principe pas commencé à courir contre eux. Comme cela revenait à dire que le contrat pouvait à tout instant être remis en cause, on comprend que la décision du Conseil d'État de mettre fin à cette situation se rattache au principe de sécurité juridique, dès lors qu'elle permet d'éviter qu'un contrat en soi valable puisse être annulé à tout instant : en faisant en sorte que sa signature purge les vices qui pouvaient affecter les actes qui s'en détachent.

D’un autre côté, la seconde exigence dérivée de la garantie des droits - le droit des administrés à un recours effectif contre les décisions qui leur sont opposées - a contraint le Conseil d'État à compenser cette évolution en ouvrant aux tiers évincés un double droit d'action contre la convention elle-même. C'est ce qui explique que les concurrents malheureux puissent désormais saisir le juge des référés aux fins de le voir ordonner la suspension de l'exécution du contrat ${ }^{48}$ tout en demandant au juge de plein contentieux de l'annuler et / ou de les indemniser du préjudice que leur a causé sa signature ${ }^{49}$.

Les implications du droit à un recours effectif apparaissent en l'espèce toutefois secondes par rapport à celles qui sont liées à l'exigence de sécurité juridique: car elles ne sont que la conséquence de la volonté du Conseil d'État de simplifier les règles applicables en la matière, de façon à tenir compte des aspirations des justiciables à une plus grande stabilité du droit.

Cette considération explique que le juge se soit efforcé de circonscrire la portée de la création de ces nouveaux recours, afin de limiter ses inconvénients. Sa crainte de fragiliser les relations contractuelles l'a en effet conduit à limiter doublement le champ d'application de ces nouvelles voies d'action. D'une part, en enfermant la possibilité de les former dans un délai de deux mois suivant l'accomplissement des mesures de publicité permettant de faire connaître l'existence de l'engagement aux tiers - la haute juridiction mettant ainsi une obligation générale inédite de publicité des contrats à la charge de l'administration. D'autre part, en réservant la possibilité de saisir le juge du contrat aux concurrents évincés de sa passation, contrairement aux conclusions du commissaire du gouvernement Didier Casas ${ }^{50}$.

42. CE, Sect., 7 octobre 1994, Epx Lopez, RFDA, 1994.1090, concl. R. Schwartz.

43. Cf. CJA, art. L. 911-1; L. 911-4 et CE, 10 décembre 2003, Institut de recherche pour le développement, AJDA, 2003.394, note J.-D. Dreyfus.

44. CJA, art. L. 911-3; L. 911-4.

45. Sur cette question, voir L. Markus et L. Perrin, annulation de l'acte détachable du contrat et distinction des contentieux, $D A, 2006, \mathrm{n}^{\circ}$ 1.

46. Note F. Raynaud et P. Fombeur sous CE, 30 octobre 1998, Ville de Lisieux, AJDA, 1998.969.

47. La solution semble d'autant plus certaine que dans ses conclusions sur cette affaire, le commissaire du gouvernement Didier Casas insistait sur le fait que «le recours pour excès de pouvoir contre l'acte détachable ne [...] répond[ait] pas aux exigences minimales [...] de sécurité juridique que l'on peut attendre aujourd'hui de» la «jurisprudence» administrative (Revue du droit public, 2007, p. 1413).

48. CJA, art. L. 521-1.

49. Sur ce point, le Conseil d'État a préféré suivre le commissaire du gouvernement Didier Casas (Revue du droit public, 2007, p. 1422) plutôt que la majorité des auteurs de doctrine, ceux-ci préconisant - depuis la seconde moitié du XX siècle au moins - de permettre aux tiers de saisir le juge de l'excès de pouvoir pour obtenir l'annulation du contrat. Voir, notamment, G. Péquignot, Théorie générale du contrat administratif, Perpignan, Impr. du Midi, 1945, p. 601 ; note D. Chauvaux et T.-X. Girardot sous CE, 10 juillet 1996, Cayzeele, AJDA, 1996.732.

50. Dans ses conclusions, l'intéressé s'était prononcé en faveur de l'ouverture de ce droit d'action aux tiers pouvant «se prévaloir d'un droit de nature patrimoniale qui aurait été lésé par la conclusion du contrat ». " Dans notre esprit », expliquait-il, « cela viserait les entreprises évincées de la procédure d'attribution d'un contrat, les usagers du service public en tout cas lorsqu'est en cause une délégation de service public ou un marché public de service public, ainsi que, peut-être, le contribuable local qui pourrait éventuellement se prévaloir de ce que les conditions financières d'un contrat ont des répercussions nécessaires sur ses droits patrimoniaux». Cette solution demeurait toutefois restrictive, dans la mesure où elle excluait «les tiers sans revendication patrimoniale tels que, notamment, les membres des assemblées délibérantes des collectivités publiques, les syndicats, les associations » (Revue du droit public, 2007, p. 1424). 
Si la première de ces réserves se comprend aisément, la seconde paraît plus contestable, dans la mesure où elle complique le droit applicable en obligeant à faire une nouvelle distinction entre les concurrents évincés et les autres tiers. De ce point de vue, la décision peut sembler contreproductive, puisqu'elle brouille la lisibilité de la règle de droit. Et on peut se demander s'il n'eût pas été préférable de reconnaître à l'ensemble des tiers justifiant d'un intérêt suffisant la faculté de contester la validité d'un contrat, après sa signature, devant le juge de plein contentieux. Dès lors en effet que la plupart d'entre eux conserve la possibilité de contester les actes détachables préalables au contrat, il semblerait plus judicieux d'étendre la solution à tous les tiers intéressés, sachant que la brièveté du délai dans lequel ils peuvent agir paraît suffisante pour prévenir le risque d'insécurité juridique. Sans doute le délai de recours sera-t-il illimité lorsque, comme en l'espèce, le contrat ne fera pas l'objet de mesures de publicité suffisantes. Mais cette exception à la règle semble justifiée au regard de l'adage nemo auditur propriam turpitudinem suam allegans (Nul n'est fondé à se prévaloir de sa propre turpitude).

La prudence est toutefois de mise, s'agissant d'un principe délicat à manipuler et qui, utilisé à mauvais escient, pourrait précariser davantage la situation des administrés au lieu de la sécuriser. D’ailleurs la consécration du principe de sécurité juridique ne conduit-elle pas le juge à accroître ses pouvoirs de façon anormale?

\section{La sécurité juridique, facteur d'un accroissement des pouvoirs du juge}

La consécration du principe de sécurité juridique semble parallèlement conduire les magistrats à accroître leurs prérogatives. Outre qu'elle a encouragé les juridictions suprêmes à se reconnaître la faculté de moduler dans le temps les effets de leurs revirements de jurisprudence (A), elle a en effet incité le Conseil d'État à donner au juge de plein contentieux le pouvoir de moduler les conséquences de l'annulation d'un contrat administratif (B).

\section{A. La reconnaissance du pouvoir des juridictions suprêmes de moduler dans le temps les effets de leurs revirements de jurisprudence}

La consécration du principe de sécurité juridique a conduit le Conseil d'État à rejeter l'applicabilité de l'un des revirements qu'il opère aux situations qui lui sont antérieures, « sous réserve des actions en justice [...] engagées » avant qu'il ne soit rendu public. Or, cette précision est loin d'être anodine. Car elle revient implicitement à dire que le renforcement des garanties accordées aux jus- ticiables passe par celui des pouvoirs du juge suprême de l'ordre administratif, ce dernier se réservant le droit d'apprécier dans le temps les effets de ses changements d'interprétation.

Cette solution confirme sa volonté de s'aligner sur la jurisprudence de la Cour de cassation, dans la mesure où cette dernière s'est reconnu la même possibilité ${ }^{51}$. Sans doute les parties pourront-elles inviter les magistrats à faire usage de ce pouvoir dans un sens ou dans un autre. Car, même si cette faculté ne leur est pas explicitement reconnue, rien ne les empêchera de l'exercer. Mais comme elles ne pourront se substituer aux juges quant au sens de la décision finale, c'est bien aux hautes cours elles-mêmes qu'il reviendra de déterminer la portée de leurs revirements.

Afin de minimiser l'ampleur de cette évolution, le Conseil d'État a cherché à en limiter la portée. C'est ainsi que son communiqué de presse tend à la circonscrire aux revirements qui intéressent «l'existence et les modalités d'exercice des recours juridictionnels eux-mêmes ${ }^{52}$. Si elle peut s'expliquer par le souci de la haute juridiction de ne pas fragiliser la stabilité des relations juridiques en multipliant les hypothèses dans lesquelles les effets de ses revirements peuvent être modulés dans le temps, cette réserve ne paraît pas justifiée à la lecture de l'arrêt. D'autant que le parallèle avec la jurisprudence judiciaire montre que la modulation a vocation à s'étendre à l'ensemble des changements d'interprétation.

Ceux-ci semblent donc voués à être construits en deux temps ${ }^{53}$ puisque, après avoir précisé la nouvelle règle applicable, le juge devra se prononcer sur sa portée temporelle. Trois hypothèses sont envisageables.

La première, qui constitue le droit commun de la matière, correspond à l'état du droit antérieur. C'est celle où la jurisprudence s'applique $a b$ initio, rétroactivement. Au contraire, les deux dernières constituent des solutions dérogatoires. L'une parce qu'elle permet au juge de ne rendre ses revirements applicables que pour l'avenir, le nouvel état du droit ne devant régir que les situations juridiques nées après son adoption; l'autre, à cheval entre les deux, lui permettant, comme en l'espèce, de rendre le revirement applicable aux situations antérieurement existantes, chaque fois qu'elles ont donné lieu à une action en justice préalablement à son intervention.

Si ce pouvoir de modulation apparaît plus en phase avec les attentes des justiciables, il soulève de graves problèmes théoriques quant à la place du juge au sein des institutions. Dès lors que l'énoncé de la nouvelle jurisprudence n'est pas nécessaire à la résolution du litige, il est en effet tentant de l'analyser comme un obiter dictum (une incidence inutile), dont la seule finalité est de consacrer une règle générale et impersonnelle valable pour l'avenir, en violation de l'article 5 du Code civil qui proscrit les « arrêts de règlement ${ }^{54}$. La faculté des juridictions

54. En ce sens, voir F. Pollaud-Dulian, «À propos de la sécurité juridique», RTD civ., 2001.502; A. Bolze, «La norme jurisprudentielle et son revirement en droit privé », $R R J, 1997.873, \mathrm{n}^{\circ}$ 26. Voir également J. Rivero, « Sur la rétroactivité de la règle jurisprudentielle », AJDA, 1968.16. 16. 
de moduler dans le temps leurs revirements de jurisprudence accréditerait ainsi l'idée de l'avènement d'un «gouvernement des juges ${ }^{55}$, le principe de sécurité juridique encourageant les magistrats à s'ériger en colégislateurs ${ }^{56}$.

Malgré son apparente rigueur, cette conclusion apparaît toutefois hâtive. Car que signifie cette expression? Si elle veut dire que le risque existe de voir les magistrats se substituer au pouvoir exécutif, on ne voit pas trop en quoi la reconnaissance de leur pouvoir de moduler les effets de leurs revirements dans le temps étaie ce reproche. S’il faut la comprendre comme dénonçant d'une façon plus générale la propension des juges à se substituer aux «élus » du peuple dans la direction des affaires publiques, la critique n'apparaît pas davantage fondée. Car leur pouvoir de création normatif ne suffit pas à faire d'eux des « décideurs publics ${ }^{57}$. Sans doute le caractère général et impersonnel de la règle jurisprudentielle évoque-t-il la loi ou le règlement. Mais plutôt que de souligner les ressemblances qui les rapprochent, il convient d'insister sur les différences qui les opposent. Car ce qui caractérise lesdits « décideurs ", c'est avant tout leur pouvoir d'initiative. C'est le fait qu'il leur revienne de déterminer le contenu du texte à adopter et le moment de le prendre. Or, les juges n'ont pas la même liberté d'action sur l'un et l'autre de ces points. De même qu'ils ne peuvent en principe donner leur interprétation de la règle de droit qu'à condition d'être saisis de la question par les parties, ils ne peuvent normalement statuer que dans les limites de l'instance. Leur liberté d'appréciation est d'ailleurs d'autant moins étendue en l'espèce qu'ils ne pourront prendre leur décision qu'après avoir scrupuleusement mis en balance les intérêts en présence, l'intérêt général d'une part, et celui des parties d'autre part. Ce n'est en effet qu'après en avoir fait le bilan que les juges suprêmes pourront déterminer les conséquences, sur le plan temporel, de leur nouvelle interprétation.

Reste à savoir si le revirement commenté est ou non légitime. La réponse à cette question passe par ce constat: le pouvoir politique peut toujours avoir le dernier mot sur les magistrats. La loi anti-Perruche ${ }^{58}$ est là pour le rappeler. Quid de l'attitude des élus consistant à critiquer celle des juges devant les médias sans la remettre en cause devant l'hémicycle? Qui ne dit mot consent. Et la classe politique doit être présumée avoir tacitement accepté une décision de justice qu'elle ne renverse juridiquement pas. Vue sous cet angle, la jurisprudence tire sa légitimité de la caution implicite que le législateur lui apporte. À la réflexion, cela n'est guère surprenant. D'abord, parce que la jurisprudence doit son existence à la loi, l'article $4 \mathrm{du}$ Code civil interdisant au juge de refuser de statuer sous prétexte de son silence. Ensuite, parce qu'un changement d'interprétation est souvent impulsé par le législateur luimême, quoique de façon indirecte. La loi n'étant légitime qu'autant quelle exprime la volonté générale, les gouvernants doivent normalement prendre acte de ses dernières aspirations. Le plus souvent ils le font en votant de nouvelles lois. Mais lorsqu'ils les adoptent sans abroger les dispositions antérieures qui leur sont contraires, le juge ne peut faire autrement que de les interpréter à la lumière des textes les plus récents, de façon à préserver l'équilibre général de l'ordonnancement juridique. Afin de découvrir leur signification nouvelle, les magistrats doivent ainsi repenser les normes litigieuses, non pas en se tenant à leur simple lettre, mais en les envisageant dans leurs rapports avec l'ensemble du corpus juridique, de façon à aboutir à une solution globalement cohérente. Or, n'est-ce pas ce que fait le juge en l'espèce? On peut se poser la question, dès lors que sa décision ne semble que tirer les conséquences, au regard de la jurisprudence, de la volonté du législateur de limiter dans le temps les effets des décisions préjudiciables aux droits acquis des particuliers, volonté qui se déduit du vote des lois du 8 février $1995^{59}$ et du 30 juin $2000^{60}$

Ainsi perçu, le pouvoir de modulation du juge, loin de constituer un obiter dictum, n'est que la manifestation de son pouvoir de juris dictio, celui-ci lui imposant de vérifier quelles sont les règles applicables au litige qui lui est soumis avant de le trancher. Dans une telle perspective, il semble en effet normal qu'il commence par énoncer quelle est la norme présentement applicable, avant de vérifier si elle l'est au cas d'espèce, compte tenu de la date des faits qui en sont à l'origine; et que, dans la négative, il lui revienne de rechercher quelle est la règle antérieure qu'il convient de mettre en œuvre.

Ainsi compris, cet aspect de la décision n'est pas aussi révolutionnaire qu'on pourrait le croire, dès lors qu'il ne fait que tirer les implications nouvelles de la consécration du principe de sécurité juridique en droit administratif. Celles-ci semblent également expliquer la reconnaissance du pouvoir du juge de plein contentieux de moduler les effets de l'annulation d'un contrat administratif.

55. Selon le mot d'Édouard Lambert (Le Gouvernement des juges et la lutte contre la législation sociale aux États-Unis, rééd. Paris, Dalloz, 2005).

56. Sur cette question, voir notamment P. Therry, «À propos d'un arrêt sur les revirements de jurisprudence ou comment faire une omelette sans casser les œufs... ", RTD civ., 2005.176 ; M. Pinault, «Incertitude et sécurité juridique », http://www.courdecassation.fr; note C. Landais et F. Lenica sous CE, 11 mai 2004, ibid., p. 1183 ; B. Seiller, « Partie ou fin de partie? », AJDA, 2006.681.

57. Pour une proposition de définition de cette expression, voir F. Bottini, La Protection des décideurs publics face au droit pénal, Thèse de doctorat, Université du Havre, 2006; Paris, LGDJ (Bibliothèque constitutionnelle et de science politique ; 130), 2008.

58. Cf. L. n ${ }^{\circ}$ 2002-303 du 4 mars 2002, relative aux droits des malades et à la qualité du système de santé, JORF, 5 mars 2002, p. 4118, art. $1^{\text {er }}$ (codifié à l'article L. 114-5 du Code de l'action sociale et des familles par la L. $\mathrm{n}^{\circ}$ 2005-102 du 11 février 2005, pour l'égalité des droits et des chances, la participation et la citoyenneté des personnes handicapées, JORF, 12 février 2005, texte $\mathrm{n}^{\circ}$ 1, art. 2) et Cass., Ass. plén., 17 novembre 2000, D., 2000 , jur., p. 332.

59. L. modifiée $\mathrm{n}^{\circ} 95-125$, relative à l'organisation des juridictions et à la procédure civile, pénale et administrative, JORF, 9 février $1995, \mathrm{p}$. 2175, art. 62 sq. codifiés dans le Code de la justice administrative par l'Ord. ${ }^{\circ}$ 2000-387 du 4 mai 2000, JORF, 7 mai 2000, p., art. 1.

60. L. modifiée $n^{\circ}$ 2000-597, relative au référé devant les juridictions administratives, JORF, $1^{\text {er }}$ juillet 2000, p. 9948. 


\section{B. La reconnaissance du pouvoir du juge de plein contentieux de moduler les effets de l'annulation d'un contrat administratif}

La consécration du principe de sécurité juridique a enfin conduit le Conseil d'État à accroître les pouvoirs du juge de plein contentieux en lui reconnaissant la faculté «d'annuler, totalement ou partiellement» un contrat administratif à la demande d'un tiers évincé de sa signature, «le cas échéant avec un effet différé », lorsque «l'annulation $[\ldots]$ porterait $[. .$.$] une atteinte excessive à l'intérêt$ général ou aux droits des cocontractants ».

Pour mesurer toute la portée de cette évolution, il convient de rappeler que l'annulation d'un contrat est en principe rétroactive. Par analogie avec la nullité que peut prononcer le juge de l'excès de pouvoir ${ }^{61}$, cette solution semble être un héritage de l'Ancien Régime. Le « juge administratif » étant alors considéré comme un supérieur hiérarchique de l'autorité cocontractante, l'annulation du contrat équivalait sous l'ancien droit à la décision de retirer celle de le passer. C'est pourquoi elle revêtait un caractère rétroactif. La Révolution n'ayant pas remis en cause cette approche de la matière, le contrat annulé est en principe censé n'avoir jamais existé ${ }^{62}$, les cocontractants étant en conséquence normalement obligés de remettre les choses dans l'état dans lequel elles se trouvaient avant sa passation ${ }^{63}$. Lorsque ce n'est matériellement pas possible, le juge administratif ordonne l'indemnisation de la partie lésée par le cocontractant, soit sur le fondement d'une responsabilité pour faute, soit sur celui de l'enrichissement sans cause ${ }^{64}$. Mais, cette alternative mise à part, le juge de plein contentieux n'a en principe pas d'autre choix que de constater avec effet immédiat l'inexistence rétroactive de la convention.

La présente décision tend à assouplir la rigueur de cette solution, en augmentant le pouvoir du juge du contrat de moduler les effets de l'annulation.

D'un point de vue matériel, il gagne la faculté d'annuler tout ou partie du contrat, de remettre en cause son existence même ou simplement celles de ses dispositions qui en sont divisibles : alors que jusqu'à présent il ne pouvait en principe que constater la nullité de la convention à la demande des parties, il pourra désormais l'annuler de sa propre initiative s'il estime que les intérêts en présence l'exigent.
D'un point de vue temporel, il conserve le droit d'en prononcer l'annulation $a b$ initio (de façon rétroactive) tandis que celui de la prononcer ex nunc (de façon immédiate; à compter du prononcé de la décision) se trouve conforté ${ }^{65}$ et qu'il gagne celui de la prononcer pro futuro, pour l'avenir, la nullité ne devenant alors effective qu'à une date ultérieure. Alors que dans le premier cas, l'annulation s'apparente à un « retrait » en raison de son caractère rétroactif, dans le deuxième elle évoque une « abrogation » en raison de son caractère immédiat, la troisième formule représentant un cas de figure inédit, sui generis pourrait-on dire. Ses nouvelles prérogatives sont d'autant plus importantes que rien ne semble interdire au juge de donner un effet temporel différent aux annulations matériellement prononcées; de rendre rétroactive la nullité de certaines dispositions du contrat, immédiate celles de certaines autres, et de différer celle d'une troisième série d'articles. Ici aussi, c'est au juge qu'il revient de déterminer, éventuellement à la demande des parties, la modulation qui paraît le plus appropriée au cas d'espèce dont il est saisi, après avoir fait le bilan des intérêts en présence, l'intérêt général devant l'emporter sur celui des cocontractants, sauf s'il ne s'oppose justement pas à ce qu'on assure la sauvegarde de leurs droits acquis.

Comme on l'a vu ${ }^{66}$ cette évolution n'est pas véritablement surprenante, puisqu'elle se trouvait elle aussi annoncée par les décisions du juge de l'excès de pouvoir de limiter le retrait des actes administratifs illégaux créateurs de droit ${ }^{67}$ et de moduler dans le temps l'annulation d'un acte administratif unilatéral ${ }^{68}$. Mais, là encore, c'est la consécration du principe de sécurité juridique dans l'ordre interne qui semble avoir décidé le Conseil à accroître les pouvoirs du juge de plein contentieux. Sans doute la haute juridiction n'a-t-elle pas clairement fondé son revirement sur cette exigence. Mais, outre qu'elle se déduit des conclusions du commissaire du gouvernement ${ }^{69}$, cette justification apparaît implicite à la lumière de la jurisprudence européenne. Car, comme la CJCE, la Cour de Strasbourg considère depuis longtemps que c'est au «principe de sécurité juridique» que les autorités doivent de pouvoir se « dispense[r] [...] de remettre en cause des actes ou situations juridiques antérieurs au prononcé » d'une annulation $^{70}$.

L'extension des pouvoirs du juge du contrat ne semble ainsi constituer qu'une autre implication nouvelle de la

61. En ce sens, voir note C. Landais et F. Lenica sous CE, 11 mai 2004, AJDA, 2004, p. 1183.

62. CE Sect. 27 novembre 1942, Société Bongrand et Dupin, rec., p. 335.

63. TA, Paris 21 avril 1971, Ville de Paris c/ Sieurs Ribette et Manoury et dame Ropert, AJDA, 1972, p. 164.

64. CE, 15 juillet 1959, Sieur Vauzelle, rec., p. 466.

65. TC, 6 mai 2002, Binet, Rec., p. 545.

66. Supra I, A.

67. CE, 26 octobre 2001, AJDA, 2001, p. 1034

68. CE, 11 mai 2004, AJDA, 2004, p. 1183.

69. Aux yeux de Didier Casas en effet, il convenait de renforcer « les pouvoirs du juge» de plein contentieux «saisi d'un recours des tiers» afin de faire en sorte que cette saisine « soit un instrument efficace, permettant aux tiers de contester la validité du contrat pour faire valoir leurs droits, tout en garantissant du mieux possible les droits des parties» (Revue du droit public, 2007, p. 1425).

70. CEDH, 13 juin 1979, Marckx c/ Belgique, série A, $n^{\circ} 31, \S n^{\circ} 58$. Dans le même ordre d'idées, voir CJCE, 8 avril 1976, Defrenne c/ Sabena, aff. 43/75, rec., p. 482 , pt 74 . 
consécration de ce principe en droit interne, celle-ci conduisant le juge administratif à accroître ses prérogatives afin de limiter « les effets déstabilisateurs des décisions de justice sur les situations juridiquement constituées ${ }^{71}$. De ce point de vue, cet aspect de la décision commentée paraît également légitime, dès lors qu'il apparaît cohérent avec la volonté du législateur d'assurer un meilleur respect des droits acquis des justiciables, afin de prendre davantage en compte leurs aspirations à une plus grande stabilité du droit.

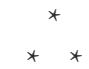

Si nombre d'auteurs se sont effrayés de la perspective de la consécration du principe de sécurité juridique en droit français, l'arrêt rendu par le Conseil d'État le 16 juillet 2007 montre qu'elle peut être source de progrès pour les particuliers. Car elle permet de faire en sorte que des changements indépendants de leurs volontés ne bouleversent par trop brutalement leur situation juridique, en leur donnant le temps de s'adapter au nouvel état du droit. Ce principe explique ainsi certaines des mutations qui affectent le droit administratif français. Il permet notamment de comprendre l'intérêt nouveau des autorités pour l'après jugement, entre autres illustré par l'adoption des lois précitées du 8 février 1995 et du 30 juin 2000 et la jurisprudence «AC! ${ }^{72}$; de même qu'il explique en partie le rapprochement actuel des recours pour excès de pouvoir et de plein contentieux.
La prudence reste toutefois de mise tant son caractère protéiforme incite à la méfiance. Deux risques de dérive semblent en effet à éviter, si l'on ne veut pas en faire une bombe à retardement.

Le premier, juridique, tient au danger de statisme du droit auquel pourrait conduire son usage outrancier. Chaque nouveau revirement de jurisprudence s'en réclamant ne devrait ainsi se faire qu'au terme d'une sorte d'étude d'impacts, permettant de mesurer les conséquences du revirement précédent sur l'état du droit. Car chaque nouvelle décision rendue sur son fondement soulève autant de questions qu'elle en résout.

Le second danger à prévenir est plus politique. Il réside dans cette crainte de voir les juges tirer profit du développement de ce principe pour s'arroger davantage de pouvoirs. Pour certains en effet, au-delà des questions proprement juridiques, «ce qui est en cause n'est rien d'autre que la forme démocratique de notre mode de gouvernement ${ }^{73}$. De ce point de vue, la décision commentée invite à réfléchir sur la relation «État de droit / démocratie». Les notions sont-elles par essence antinomiques? Ou bien est-il possible de les concilier, en faisant de la première l'instrument de la seconde ${ }^{74}$ ? Si oui, la présente décision ne participe-t-elle pas d'un vaste rééquilibrage devant permettre au juge de jouer pleinement son rôle de contre-pouvoir, afin de renforcer les garanties offertes aux gouvernés contre l'arbitraire des gouvernants? Autant de questions qui laissent présager de nouvelles évolutions du droit administratif.

71. Ibid.

72. Celles-ci limitant dans le temps les effets des décisions préjudiciables aux droits acquis des particuliers.

73. V. Heuzé, «À propos du rapport sur les revirements de jurisprudence. Une réaction entre indignation et incrédulité », JCP G, $2005, \mathrm{I}_{130} \mathrm{n}^{\circ}{ }^{12}$.

74. Sur cette question, voir F. Bottini, «La promulgation des lois parlementaires », RFDC, $\mathrm{n}^{\circ}$ 76, 2008, p. 761. 


\section{ANNEXE \\ CE, Ass., 16 juillet 2007, Société Tropic Travaux Signalisation}

Vu la requête, enregistrée le 21 mars 2006 au secrétariat du contentieux du Conseil d'État, présentée pour la SOCIÉTÉ TROPIC TRAVAUX SIGNALISATION ; la SOCIÉTÉ TROPIC TRAVAUX SIGNALISATION demande au Conseil d'État:

$1^{\circ}$ ) d'annuler l'ordonnance du 2 mars 2006 par laquelle le juge des référés du tribunal administratif de Basse-Terre, statuant en application de l'article L. 521-1 du code de justice administrative, a rejeté sa demande tendant à la suspension de la décision en date du 14 novembre 2005 de la chambre de commerce et d'industrie de Pointe-à-Pitre rejetant son offre pour le marché de marquage des aires d'avions de l'aéroport Le Raizet à Pointe-à-Pitre, de la décision d'attribuer ce marché à l'entreprise Rugoway, de la décision de signer ce marché et du marché lui-même;

$2^{\circ}$ ) statuant sur le fondement des dispositions de l'article L. 521-1 du code de justice administrative, de faire droit à la demande de suspension présentée devant le tribunal administratif de Basse-Terre;

Vu les autres pièces du dossier;

Vu le code de justice administrative, notamment ses articles R. 122-17, R.122-18 et R. 611-20;

Après avoir entendu en séance publique :

- le rapport de $\mathrm{M}^{\mathrm{me}}$ Nathalie Escaut, Maître des Requêtes,

- les observations de la SCP Waquet, Farge, Hazan, avocat de la SOCIÉTÉ TROPIC TRAVAUX SIGNALISATION et de la SCP Richard, avocat de la chambre de commerce et d'industrie de Pointe-à-Pitre,

- les conclusions de M. Didier Casas, Commissaire du gouvernement;

Considérant qu'il ressort des pièces du dossier soumis au juge des référés qu'après avoir été informée, le 14 novembre 2005, par la chambre de commerce et d'industrie de Pointe-à-Pitre du rejet de l'offre qu'elle avait présentée pour l'attribution d'un marché portant sur le marquage des aires d'avions et des chaussées routières de l'aéroport de Pointe-à-Pitre le Raizet, la SOCIÉTÉ TROPIC TRAVAUX SIGNALISATION a saisi le juge des référés du tribunal administratif de Basse-Terre, sur le fondement des dispositions de l'article L. 521-1 du code de justice administrative, d'une demande tendant à la suspension de l'exécution de ce rejet de son offre, de la décision de la chambre de commerce et d'industrie acceptant l'offre de la société Rugoway, de sa décision de signer le marché et du marché lui-même ; que par une ordonnance en date du 2 mars 2006, à l'encontre de laquelle la SOCIÉTÉ TROPIC TRAVAUX SIGNALISATION se pourvoit en cassation, le juge des référés a rejeté cette demande;

Sans qu'il soit besoin d'examiner l'autre moyen de la requête ;

Considérant que, indépendamment des actions dont les parties au contrat disposent devant le juge du contrat, tout concurrent évincé de la conclusion d'un contrat administratif est recevable à former devant ce même juge un recours de pleine juridiction contestant la validité de ce contrat ou de certaines de ses clauses, qui en sont divisibles, assorti, le cas échéant, de demandes indemnitaires; que ce recours doit être exercé, y compris si le contrat contesté est relatif à des travaux publics, dans un délai de deux mois à compter de l'accomplissement des mesures de publicité appropriées, notamment au moyen d'un avis mentionnant à la fois la conclusion du contrat et les modalités de sa consultation dans le respect des secrets protégés par la loi ; qu'à partir de la conclusion du contrat, et dès lors qu'il dispose du recours ci-dessus défini, le concurrent évincé n'est, en revanche, plus recevable à demander l'annulation pour excès de pouvoir des actes préalables qui en sont détachables;

Considérant que, ainsi saisi de telles conclusions par un concurrent évincé, il appartient au juge, lorsqu'il constate l'existence de vices entachant la validité du contrat, d'en apprécier les conséquences; qu’il lui revient, après avoir pris en considération la nature de l'illégalité éventuellement commise, soit de prononcer la résiliation du contrat ou de modifier certaines de ses clauses, soit de décider de la poursuite de son exécution, éventuellement sous réserve de mesures de régularisation par la collectivité contractante, soit d'accorder des indemnisations en réparation des droits lésés, soit enfin, après avoir vérifié si l'annulation du contrat ne porterait pas une atteinte excessive à l'intérêt général ou aux droits des cocontractants, d'annuler, totalement ou partiellement, le cas échéant avec un effet différé, le contrat; que, par ailleurs, une requête contestant la validité d'un contrat peut être accompagnée d'une demande tendant, sur le fondement des dispositions de l'article L. 521-1 du code de justice administrative, à la suspension de son exécution;

Considérant qu'il appartient en principe au juge d'appliquer les règles définies ci-dessus qui, prises dans leur ensemble, n'apportent pas de limitation au droit fondamental qu'est le droit au recours; que toutefois, eu égard à l'impératif de sécurité juridique tenant à ce qu'il ne soit pas porté une atteinte excessive aux relations contractuelles en cours et sous réserve des actions en justice ayant le même objet et déjà engagées avant la date de lecture de la présente décision, le recours ci-dessus défini ne pourra être exercé qu'à l'encontre des contrats dont la procédure de passation a été engagée postérieurement à cette date ;

Considérant qu'en rejetant comme irrecevables les conclusions de la SOCIÉTÉ TROPIC TRAVAUX SIGNALISATION à fin de suspension du marché conclu entre la chambre de commerce et d'industrie de Pointe-à-Pitre et la société Rugoway, sans rechercher si la SOCIÉTÉ TROPIC TRAVAUX SIGNALISATION s'était portée candidate à l'attribution de ce marché, le juge des référés du tribunal administratif de Basse-Terre a commis une erreur de droit entachant le bien-fondé de l'ensemble de son ordonnance ; 
Considérant qu'il résulte de qui précède que la SOCIÉTÉ TROPIC TRAVAUX SIGNALISATION est fondée à demander l'annulation de l'ordonnance attaquée du juge des référés du tribunal administratif de Basse-Terre;

Considérant qu'il y a lieu, pour le Conseil d'État, par application de l'article L.821-2 du code de justice administrative, de régler l'affaire au titre de la procédure de référé engagée;

Considérant qu’aux termes de l'article L. 521-1 du code de justice administrative: «Quand une décision administrative, même de rejet, fait l'objet d'une requête en annulation ou en réformation, le juge des référés, saisi d'une demande en ce sens, peut ordonner la suspension de l'exécution de cette décision, ou de certains de ses effets, lorsque l'urgence le justifie et qu'il est fait état d'un moyen propre à créer, en l'état de l'instruction, un doute sérieux quant à la légalité de la décision [...] »;

Considérant qu'il résulte de l'instruction que le délai de recours contre le marché conclu entre la chambre de commerce et d'industrie de Pointe-à-Pitre et la société Rugoway n'ayant pas couru faute de mesure de publicité appropriée, la SOCIÉTÉ TROPIC TRAVAUX SIGNALISATION, en sa qualité de concurrent évincé de l'attribution de ce marché, est recevable à demander la suspension de son exécution sur le fondement des dispositions de l'article L. 521-1 du code de justice administrative; que toutefois, en l'état de l'instruction, le seul moyen d'annulation qu'elle soulève et qui est tiré du détournement de pouvoir, n'est pas de nature à faire naître un doute sérieux quant à la légalité de ce marché; que, par suite, ses conclusions tendant à la suspension de son exécution doivent être rejetées;

Considérant que, compte tenu de la signature du marché contesté le 26 novembre 2005, la société requérante n'était plus recevable à la date de l'introduction de sa demande, le 13 janvier 2006, à demander l'annulation pour excès de pouvoir des actes préalables qui en sont détachables; que dès lors, ses conclusions à fin de suspension des décisions de la chambre de commerce et d'industrie rejetant son offre, attribuant le marché à la société Rugoway et décidant de le signer ne peuvent également qu'être rejetées;

Sur les conclusions tendant à l'application des dispositions de l'article L. 761-1 du code de justice administrative :

Considérant qu'il n'y a pas lieu, dans les circonstances de l'espèce, de faire application des dispositions de l'article L. 761-1 du code de justice administrative et de mettre à la charge de la SOCIÉTÉ TROPIC TRAVAUX SIGNALISATION la somme que la chambre de commerce et d'industrie de Pointe-à-Pitre demande au titre des frais exposés par elle et non compris dans les dépens;

\section{DÉCIDE :}

Article $1^{\text {er }}$ : L’ordonnance en date du 2 mars 2006 du juge des référés du tribunal administratif de Basse-Terre est annulée.

Article 2: La requête de la SOCIÉTÉ TROPIC TRAVAUX SIGNALISATION devant le juge des référés du tribunal administratif de Basse-Terre est rejetée.

Article 3: Les conclusions de la chambre de commerce et d'industrie de Pointe-à-Pitre tendant à l'application des dispositions de l'article L. 761-1 du code de justice administrative sont rejetées.

Article 4: La présente décision sera notifiée à la SOCIÉTÉ TROPIC TRAVAUX SIGNALISATION, à la chambre de commerce et d'industrie de Pointe-à-Pitre et à la société Rugoway.

Copie pour information en sera adressée au Premier ministre, au ministre d'État, ministre de l'écologie, du développement et de l'aménagement durables et au ministre de l'économie, des finances et de l'emploi. 